\title{
Vertical Transmission of Zika Virus in Aedes aegypti Produces Potentially Infectious Progeny
}

\author{
Genevieve Comeau, ${ }^{1}$ Robert A. Zinna, ${ }^{2}$ Taylor Scott, ${ }^{1}$ Kacey Ernst, ${ }^{3}$ Kathleen Walker, ${ }^{1}$ Yves Carrière, ${ }^{1}$ and Michael A. Riehle ${ }^{1 *}$ \\ ${ }^{1}$ Department of Entomology, University of Arizona, Tucson, Arizona; ${ }^{2}$ Department of Biology, Mars Hill University, Mars Hill, North Carolina; \\ ${ }^{3}$ Department of Epidemiology and Biostatistics, Mel and Enid Zuckerman College of Public Health, University of Arizona, Tucson, Arizona
}

\begin{abstract}
Vertical transmission, or pathogen transfer from female to offspring, can facilitate the persistence of emerging arboviruses, such as Zika virus (ZIKV), through periods of low horizontal transmission or adverse environmental conditions. We aimed at determining the rate of vertical transmission for ZIKV in its principal vector, Aedes aegypti, and the vector competence of vertically infected progeny. Aedes aegypti females that consumed a blood meal provisioned with ZIKV were maintained under three temperature conditions $\left(27^{\circ} \mathrm{C}, 30^{\circ} \mathrm{C}\right.$, and $\left.33^{\circ} \mathrm{C}\right)$ following the infectious blood meal and allowed to complete three reproductive cycles. The overall vertical transmission rate was $6.5 \%(95 \% \mathrm{Cl}=3.9-9.9)$. Vertical transmission of ZIKV was observed across all temperature conditions and virus detected in adult progeny up to 2 weeks postemergence. In total, $3.4 \%(95 \% \mathrm{CI}=1.6-6.2)$ of adult progeny produced saliva with ZIKV, indicating their vector competence. These results suggest the virus may be maintained in Ae. aegypti populations without a vertebrate host for short periods.
\end{abstract}

\section{INTRODUCTION}

Zika virus (ZIKV) is a mosquito-borne flavivirus that emerged as a significant human pathogen during the 2015 pandemic. ${ }^{1}$ Although asymptomatic in approximately $80 \%$ of cases, ZIKV infection can cause adverse pregnancy outcomes, such as Zika congenital syndrome, and serious neural complications in children and adults including Guillain-Barre syndrome and fatal encephalitis. ${ }^{2,3}$ It is primarily transmitted to humans through the bite of the mosquito Aedes aegypti, although other mosquito species are also competent. ${ }^{4}$ During the outbreak in 2015, 2.2 billion people lived in areas that reported active ZIKV transmission. ${ }^{5} \mathrm{~A}$ better understanding of how ZIKV becomes permanently established after introduction to these new locales is needed to assess risk and prevent disease.

Arthropod-borne viruses, or arboviruses, can become routinely transmitted in a geographic region through several mechanisms, including 1) regular viral reintroduction and circulation across geographies, 2) establishment of mosquito and human or nonhuman reservoir transmission cycles, or 3 ) virus maintenance in the vector population. ${ }^{6}$ Maintenance in the vector population sustains viruses when there are few human or reservoir hosts, such as between outbreaks. ${ }^{7}$ It also helps arboviruses, such as Ross River and Sindbis, survive cold temperatures and low vector population numbers during harsh winter months or during dry season in monsoonal climates. ${ }^{8,9}$ Vertical transmission, or direct pathogen transfer from a female to its offspring, is one path to arboviral maintenance in vector populations. Vertical transmission of other Ae. aegypti-borne viruses such as dengue, chikungunya, and yellow fever has been documented in both the laboratory and field for Ae. aegypti. ${ }^{10-13}$ Aedes aegypti also lay desiccationresistant eggs, which greatly increases the amount of time a virus can persist. ${ }^{14}$ Interestingly, vertical transmission occurs at a higher rate in Aedes than other genera of mosquitoes. ${ }^{7}$ If $\mathrm{ZIKV}$ is also vertically transmitted, it may increase the risk of viral persistence.

*Address correspondence to Michael A. Riehle, Department of Entomology, University of Arizona, P.O. Box 210036, Forbes 41, Tucson, AZ 85721. E-mail: mriehle@ag.arizona.edu
Maintenance in the mosquito population through vertical transmission of ZIKV alone is not sufficient to maintain the virus or start another outbreak without a vertebrate host. To horizontally infect a human host, virus must be present in the saliva of the mosquito. Whether vertically infected mosquitoes produce saliva infected with ZIKV has not been evaluated. Besides creating a link between transmission cycles, vertically infected progeny with infectious saliva would largely bypass the extrinsic incubation period (EIP), which is the time between the ingestion of a viremic blood meal by a female mosquito until the virus arrives in the mosquito saliva. Estimates of the EIP of ZIKV range from 4 to 10 days, and this EIP could impact ZIKV transmission because of the limited life span of Ae. aegypti in the field. ${ }^{15-17}$ Furthermore, vertically infected mosquitoes do not need to obtain an initial ZIKV-infected blood meal, leading to younger infectious mosquitoes. If the saliva of vertically infected mosquitoes is infected with ZIKV earlier than horizontally infected mosquitoes, they would have more potential opportunities to transmit the virus.

Previous studies have implicated ZIKV as vertically transmitted, but evidence is limited by study conditions and end points. One study demonstrated a low rate (1/290 adults) of vertical transmission in adult progeny. However, virus was introduced intrathoracically into female mosquitoes, which bypasses midgut invasion and escapes barriers and could impact the rate of infection. ${ }^{18} \mathrm{~A}$ subsequent study demonstrated higher rates of vertical transmission in immature mosquitoes (1/84 larvae), but this rate might not accurately reflect infection status following adult eclosion and does not provide insights into whether ZIKV-infected adults have virus in their saliva. ${ }^{19} \mathrm{~A}$ third study found exceptionally high levels of ZIKV vertically transmitted to their progeny in both the first (34-66\% positive egg pools and $\sim 17 \%$ salivary glands) and second (52-54\% positive egg pools and $2 \%$ salivary glands) gonotrophic cycles. ${ }^{20}$ These studies did not examine how vertical transmission varies under different environmental conditions, especially because factors such as temperature and humidity conditions can strongly influence arboviral transmission dynamics. ${ }^{21-24}$ Evidence from the field also suggests that vertical transmission occurs, with two studies demonstrating that field-collected $A e$. aegypti eggs were positive for the ZIKV virus. ${ }^{25,26}$ The number of gonotrophic cycles and adult mosquito age also affect vertical transmission rates of arboviruses. ${ }^{7,20,27}$ To date, no studies have investigated 
the rate of ZIKV vertical transmission at multiple temperature conditions or how long the virus persists in adult progeny.

The objective of this study, therefore, was to quantify the rate of ZIKV vertical transmission to adult offspring of orally infected Ae. aegypti females across multiple temperature conditions, gonotrophic cycles, and adult progeny ages. As many countries reported active ZIKV transmission after the 2015 outbreak, the results of this study will help inform public health policy, surveillance strategies, and potential interventions.

\section{METHODS}

Mosquito rearing. Aedes aegypti mosquitoes, UGAL strain originating from a wild-type colony established at the University of Georgia in the early 1970s, were reared in an Arthropod Containment Level 2 insectary at $27^{\circ} \mathrm{C}, 75 \%$ relative humidity, and 16:8 light:dark photoperiod cycle. ${ }^{28}$ Larval mosquitoes were reared at a density of 100-150 larvae per liter of water and fed ground Purina Cat Chow Complete dry cat food. Pupae were transferred to $1-L$ emergence cages $(10 \times$ $6 \times 6$ inches) at a density of 50 mosquitoes/cage. Adult mosquitoes were provided with $10 \%$ sucrose ad libitum via soaked cotton balls. Sucrose-soaked cotton balls were replaced with water-soaked cotton balls 24 hours before blood meals to encourage feeding. Mosquitoes were provided with whole human blood obtained from the American Red Cross (IBC protocol \#2010-014) for propagation of the colony.

Cell culture and virus propagation. Vero cells $(\mathrm{WHO})$ were cultured in Dulbecco's modified Eagle's medium (DMEM) supplemented with $10 \%$ fetal bovine serum and incubated at $37^{\circ} \mathrm{C}$ and $5 \% \mathrm{CO}_{2}$. At $80 \%$ confluence, cells were either split 1:5 or infected with ZIKV strain PRVABC59 (BEI Resources), isolated from an infected human from Puerto Rico in December 2015. During infection culture, the medium was removed, then $2 \mathrm{~mL}$ of fresh medium was mixed with virus and diluted to a multiplicity of infection of 10 . After dilution, cells were incubated for 1 hour at $37^{\circ} \mathrm{C}$ and $5 \% \mathrm{CO}_{2}$, rocking the flask every 15 minutes. After 1 hour, the infectious medium was removed and fresh medium added to the flask. After the new medium was added, cells were incubated for 96 hours at $37^{\circ} \mathrm{C}$ and $5 \% \mathrm{CO}_{2}$ until $90 \%$ cytopathic effect was attained. Cell culture medium was then pipetted into a $15-\mathrm{mL}$ tube and centrifuged at $300 \times g$ for 10 minutes. ${ }^{29}$ Viral supernatant was transferred to a new $15-\mathrm{mL}$ tube, mixed to $20 \%$ volume/ volume with fetal bovine serum, and stored at $-80^{\circ} \mathrm{C}$ until use.

Plaque assay. Plaque assays were conducted based on protocols provided by Virapur (San Diego, CA) and those developed by Agbulos et al. ${ }^{30}$ to determine the ZIKV stock viral titer before oral infections, with slight modifications. Zika virus stock titers were also assessed using qPCR (see ZIKV qPCR methods). For plaque assays, Vero cells were plated in six-well plates and incubated overnight. The medium was removed from the cells and viral stock diluted in serum-free DMEM from $10^{-2}$ to $10^{-6}$. The ZIKV dilutions were allowed to adsorb onto cells for 1 hour, rocking every 15 minutes to distribute virus among the cells. Following adsorption, the infectious medium was removed and $3 \mathrm{~mL}$ of DMEM mixed with $4 \%$ agarose was overlaid onto the cells. The overlaid Vero cells were incubated for 5 days at $37^{\circ} \mathrm{C}$ and $5 \% \mathrm{CO}_{2}$, then stained with 0.1 volume of $5 \mathrm{mg} / \mathrm{mL}$ 3-(4,5-dimethylthiazol-2-YI)-2,5-diphenyltetrazolium bromide stain (MTT) and incubated for a minimum of 2 hours before final imaging and quantification.
Zika virus qPCR standard generation and viral qPCR quantification assay. A region of the envelope protein of ZIKV strain PRVABC59 (833 bp) was generated to quantify viral load using specific primers (forward: ATCTAGAAGAGCCGTGACGC; reverse: CTGAAAAGTCAAGGCCTGTC) which were designed to flank the qPCR amplicon primers developed by Franz et al. ${ }^{31}$ Viral RNA was extracted from the infected medium using Trizol as follows: viral supernatant (100 uL) was added to $500 \mathrm{uL}$ of Trizol. Chloroform (50 uL) was added and the sample incubated for 10 minutes at room temperature, followed by centrifugation at $4^{\circ} \mathrm{C}$ and $14,000 \mathrm{~g}$ for 15 minutes. The aqueous phase (150 $\mathrm{uL}$ ) was removed and incubated for 1 minute at room temperature with 50 $\mathrm{uL}$ of isopropanol, then centrifuged at $4^{\circ} \mathrm{C}$ and a $14000 \mathrm{~g}$ for 15 minutes. Ethanol (400 uL, $80 \%$ ) was added to each sample and incubated at room temperature for 20 minutes to evaporate, then resuspended in diethyl pyrocarbonate (DEPC) water. The isolated ZIKV total RNA was converted into cDNA using Applied Biosystem's High-Capacity cDNA kit (ThermoFisher, Waltham, MA). The target region was subsequently amplified using PCR (GoTaq, Promega, Madison, WI) and the amplicon purified using the QIAquick Gel extraction kit (Qiagen, Hilden, Germany). The purified amplicon was ligated into the pGEM-T Easy Vector (Promega), and then transfected into chemically competent E. coli (JM109). After transfected colonies were selected, the plasmid was purified using the QIAprep Spin Miniprep kit (Qiagen) and sequenced to verify the ZIKV amplicon. A T7 flanking sequence was added to the $5^{\prime}$ end using PCR, and this new PCR product was purified and used as a template to generate pure ssRNA with the MEGAscript T7 RNA synthesis kit (Ambion, ThermoFisher, Waltham, MA) according to the manufacturers' protocols. The ssRNA standard was quantified using a Nanodrop 2000 (ThermoFisher), and the concentration of RNA was used to calculate the number of ssRNA copies $(N)$ in the standard. The quantified ssRNA was diluted to generate a standard curve for absolute qPCR quantification with a concentration range of $2.13 \mathrm{E}^{+4}$ copies $-2.13 \mathrm{E}^{+8}$ copies that was incorporated into each plate.

The following primers and TaqMan probes for the detection of ZIKV with qPCR methodology were used: forward: CCGCTGCCCAACACAAG, reverse: CCACTAACGTTCTITTGCAGACAT, and probe: FAM-CTYAGACCAGCTGAAR-BBQ (26). Zika virus qPCR quantification was performed using the iTaq Universal Probes One-Step Kit (Bio-Rad, 172-5140, Hercules, $\mathrm{CA})$ according to the manufacturer's protocol with a 10-uL reaction volume. RNA extracted from uninfected female Ae. aegypti was included in each plate as a negative control. Positive controls were included using known concentrations of the ZIKV supernatant derived from the infected Vero cells. Reactions were run on an Eppendorf RealPlex2 Mastercycler for 10 minutes at $50^{\circ} \mathrm{C}, 3$ minutes at $95^{\circ} \mathrm{C}$, followed by 40 cycles of $95^{\circ} \mathrm{C}$ for 15 seconds, and $60^{\circ} \mathrm{C}$ for 30 seconds. Samples were considered positive for ZIKV if amplification was detected at or below a cycle threshold $\left(C_{t}\right)$ value of 35 , which was selected based on the average $C_{t}$ value of our negative controls (mean $=37.74 ; 95 \% \mathrm{Cl}=35.83$, 39.65) across all plates. Control $C_{t}$ values with an SD great than 10 between triplicates were manually reviewed and the outlier removed. Among all our negative controls $(n=76)$, only a single control could potentially be misidentified as a false positive. This plate had consistently low Ct values and was not included in the final analysis. This Ct threshold is quite conservative compared with that used by the CDC, which recommends a $C_{t}$ threshold value of $38 .^{32}$ Although this decreases the sensitivity of our 
analyses and results in an increased number of false negatives, given the research subject, specificity was given priority to ensure we were observing vertical transmission.

Preparation of infectious blood meal and oral infection of mosquitoes. Zika virus PRVABC59 cell supernatant was diluted to the desired concentration and mixed with whole human blood provided by the American Red Cross, taking care to ensure that the virus solution added to the blood did not exceed $10 \%$ of the total volume to minimize dilution of the blood's nutritional value. The final titer of the blood meal for both trials was $6.4 \mathrm{E}^{+7}$ viral copies $/ \mathrm{mL}$ as verified by $\mathrm{qPCR}$ and $1.2 \mathrm{E}^{+3}$ plaque-forming unit (PFU)/mL as determined by plaque assay. This concentration corresponds to the clinically observed range of ZIKV in human blood titers. ${ }^{33}$ The discrepancy between the viral copies and plaque forming units is likely because of underestimation of infectious particles by the plaque assay, which was only successful for one blood meal. Thirty 2-day-old adult female mosquitoes were transferred to small containers and allowed to feed on the ZIKVsupplemented blood from a water-jacketed membrane feeding system. After feeding for 1 hour, mosquitoes were cold anaesthetized, and female mosquitoes with visibly engorged abdomens were separated, while non-blood-fed mosquitoes were discarded. A total of 180 blood-fed female mosquitoes, 20 mosquitoes/cage, were separated into three temperature treatments of $27^{\circ} \mathrm{C}, 30^{\circ} \mathrm{C}$, and $33^{\circ} \mathrm{C}$ with 60 female mosquitoes (i.e., three cages) per treatment.

Collection of potentially vertically infected eggs. At 0 days postexposure (dpe), each cage was provided with an oviposition substrate, and female mosquitoes were allowed to oviposit freely for 72 hours, representing the first gonotrophic cycle, after which the egg sheet was removed. Egg sheets were maintained in separate, sterile plastic petri dishes for at least 72 hours to ensure the completion of embryogenesis, but not more than a month, before hatching. Female mosquitoes were provided with additional uninfected blood meals at 5 and 10 dpe to facilitate additional reproductive cycles. Oviposition substrates were provided after each blood meal, corresponding to the second and third gonotrophic cycles.

Zika virus infection and dissemination in orally infected female Ae. aegypti. At 3, 7, and 14 dpe, 20 adult female mosquitoes from each temperature treatment and gonotrophic cycle cohort were cold anesthetized to collect abdomen, leg/wing, and saliva samples. These samples represented mosquito ZIKV infection, dissemination, and infectiousness to humans, respectively. Legs and wings were removed and stored in $500 \mathrm{uL}$ of Trizol, and then each female's proboscis was inserted into a $0.2-\mathrm{mm}$ capillary tube and allowed to salivate into mineral oil for 5 minutes, after which the saliva sample was aspirated into $200 \mathrm{~mL}$ of DMEM mixed with $2 \%$ fetal bovine serum and $1 \%$ penicillin/streptomycin for processing. Saliva was stored at $-80^{\circ} \mathrm{C}$ before RNA extraction for assessing viral presence as a measure of potential infectiousness to humans. Abdomens were removed and stored in the same manner as the legs/wings. Leg/wing and abdomen samples were homogenized in 1.6- $\mathrm{mL}$ tubes with $500 \mathrm{uL}$ of Trizol, and total RNA was isolated with Trizol as described previously. For saliva samples, $100 \mathrm{uL}$ of saliva/DMEM solution was placed in $200 \mathrm{uL}$ of Trizol to inactivate any virus, then processed as before. Concentrations of the extracted RNA were verified using a Nanodrop 2000 (ThermoFisher) and stored at $-80^{\circ} \mathrm{C}$ until a viral titer could be determined by qPCR as described previously. To verify successful RNA extraction and cDNA synthesis, a qPCR was run with Ae. aegypti actin primers and an SYBR Green one-step kit according to the manufacturers' protocol (ThermoFisher).

Vertical transmission to progeny. Progeny of orally infected female mosquitoes were reared according to the methods described earlier. After adult emergence, progeny mosquitoes were maintained in cages made from sterile deli cups at 20 mosquitoes/cage. At 3, 7, and 14 days after adult emergence, 10 adult female mosquitoes from each temperature treatment and gonotrophic cycle cohort were cold anesthetized to collect and process saliva and abdomen samples as described earlier. Legs and wings were removed and discarded.

Statistical analyses. Evaluating the effects of the explanatory variables (i.e., female postinfection temperature, gonotrophic cycle, and progeny age) simultaneously using logistic regression was not possible because the sample size did not allow this type of stratification of the data and resulted in unstable estimates of the regression coefficients. Accordingly, separate univariate logistic regression models were used to evaluate the effect of 1) female postinfection temperature, 2) gonotrophic cycle, 3 ) progeny age, and 4) experimental trial ( $n=$ 2) on the odds of progeny infection. The experimental trial was treated as a categorical variable, whereas female temperature $\left(27^{\circ} \mathrm{C}, 30^{\circ} \mathrm{C}\right.$, or $\left.33^{\circ} \mathrm{C}\right)$, gonotrophic cycle (first, second, or third), and progeny age $(3,7$, or $14 \mathrm{dpe})$ were treated as numerical variables. Three outcomes were modeled: the odds of progeny with detectable virus overall, the odds of progeny with detectable virus in the saliva, and the odds of progeny with detectable virus in the abdomen. Exact binomial 95\% Cls were calculated for the percentage of ZIKV-infected mosquitoes for each level of the explanatory variables. Odds ratios reported for numerical predictors are unit odds ratios. For the categorical predictor experimental trial, the first trial was held as the reference category and the odds of the second trial relative to the first are reported. A Pearson chi-squared test with Yates's continuity correction was used to evaluate whether viral presence in the abdomen was associated with viral presence in the saliva of individual mosquitoes. The observed proportion of progeny mosquitoes with both infected abdomens and saliva was compared with the expected proportion to evaluate whether the two types of infection are independent, which was the product of the observed proportions of progeny mosquitoes with infected abdomens only and infected saliva only.

\section{RESULTS}

Orally infected Ae. aegypti females transmit ZIKV to their offspring. To assess the rate of ZIKV vertical transmission, we examined orally infected female Ae. aegypti and their offspring for viral presence at multiple time points over a 2 -week period. At least one female mosquito with disseminated ZIKV infection, indicating capacity to vertically transmit the virus to progeny, was present in every cage at the time of egg sheet collection. However, the proportion of female mosquitoes with a disseminated infection ranged from $10 \%$ to $90 \%$ of mosquitoes per cage. The average maternal infection rate, or proportion of blood-fed females with confirmed ZIKV infections, by temperature condition was $40 \%$ at $27^{\circ} \mathrm{C}, 45 \%$ at $30^{\circ} \mathrm{C}$, and $20 \%$ at $33^{\circ} \mathrm{C}$. The average maternal dissemination rate for these temperatures was $39 \%$ at $27^{\circ} \mathrm{C}, 49 \%$ at $30^{\circ} \mathrm{C}$, 
TABLE 1

Logistic regression analysis of Zika virus vertical transmission

\begin{tabular}{|c|c|c|c|c|}
\hline Explanatory variable & Progeny infection outcome & $x^{2}$ & $P$-value & Odds ratic \\
\hline \multirow[t]{3}{*}{ Experimental trial } & Total & 0.92 & 0.34 & 0.59 \\
\hline & Abdomen & 1.41 & 0.24 & 0.39 \\
\hline & Saliva & 0.11 & 0.74 & 0.79 \\
\hline \multirow{3}{*}{ Female temperature } & Total & 1.59 & 0.21 & 0.87 \\
\hline & Abdomen & 0 & 0.99 & 1 \\
\hline & Saliva & 2.72 & 0.10 & 0.75 \\
\hline \multirow[t]{3}{*}{ Gonotrophic cycle } & Total & 3.84 & 0.05 & 1.95 \\
\hline & Abdomen & 1.76 & 0.18 & 1.92 \\
\hline & Saliva & 1.19 & 0.28 & 1.76 \\
\hline \multirow[t]{3}{*}{ Progeny age } & Total & 3.91 & 0.05 & 0.87 \\
\hline & Abdomen & 4.07 & 0.04 & 0.77 \\
\hline & Saliva & 0 & 0.99 & 1 \\
\hline
\end{tabular}

reported as unit odds ratios, and odds ratios for categorical predictors are relative to the reference category. Statistically significant models $(P \leq 0.05)$ are in bold.

and $33 \%$ at $33^{\circ} \mathrm{C}$. At $18 \%$, the maternal infection rate for the first gonotrophic cycle was lower than the maternal infection rate of $55 \%$ in the second gonotrophic cycle and $57 \%$ in the third. Maternal dissemination rates demonstrated a similar pattern. The first gonotrophic cycle at $24 \%$ was comparatively lower than the second and third cycles at $59 \%$ and $50 \%$, respectively.

Overall, $6.5 \%(95 \% \mathrm{Cl}=3.9-9.9)$ of adult progeny of orally infected female mosquitoes had detectable levels of ZIKV. Vertical transmission from orally infected $A$. aegypti females to their offspring occurred across all female temperature conditions, gonotrophic cycles, and adult progeny ages tested. The experimental trial was not a significant explanatory variable in any analysis, indicating independence of progeny outcome from the experimental trial (Table 1).

Zika virus vertical transmission occurs in a range of temperature conditions. We maintained orally infected female mosquitoes at $27^{\circ} \mathrm{C}, 30^{\circ} \mathrm{C}$, and $33^{\circ} \mathrm{C}$ to investigate ZIKV vertical transmission at a range of temperature conditions. Zika virus was present in progeny of female mosquitoes maintained at all temperatures tested (Figure 1A). Zika virus was found in the abdomens of adult progeny from every temperature, and in the saliva of adult progeny from $27^{\circ} \mathrm{C}$ to $30^{\circ} \mathrm{C}$, but not from $33^{\circ} \mathrm{C}$ (Figure 1B). There was no significant association between female temperature and the odds of ZIKV infection in the progeny mosquitoes (Table 1).

\section{ZIKV Vertical Transmission by Maternal Temperature}

\section{A By Individual Progeny}

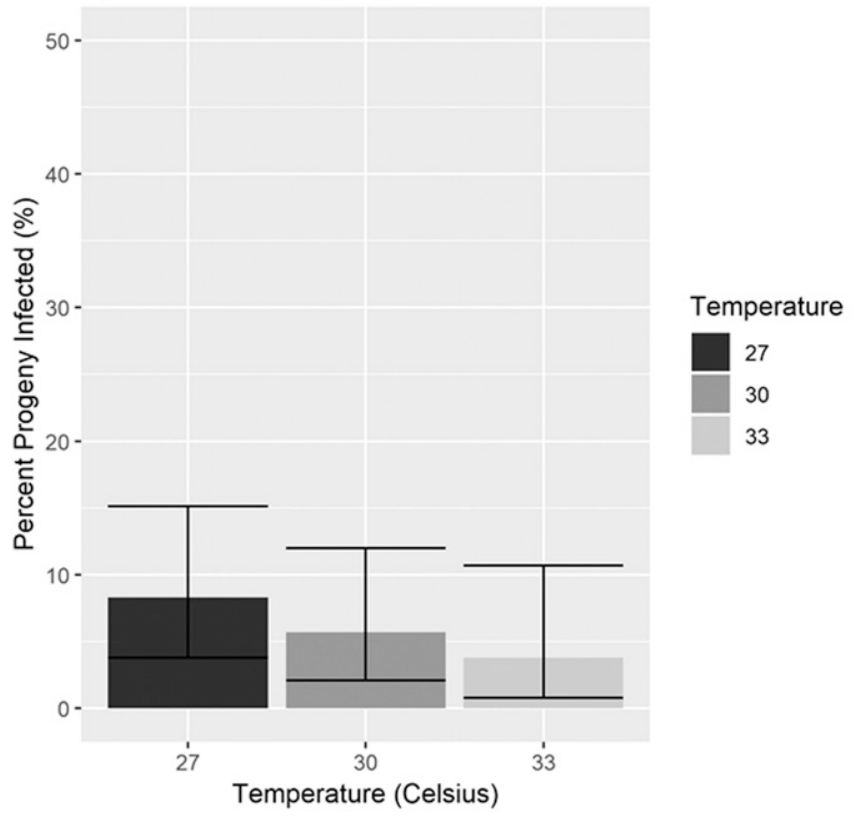

B By Sample

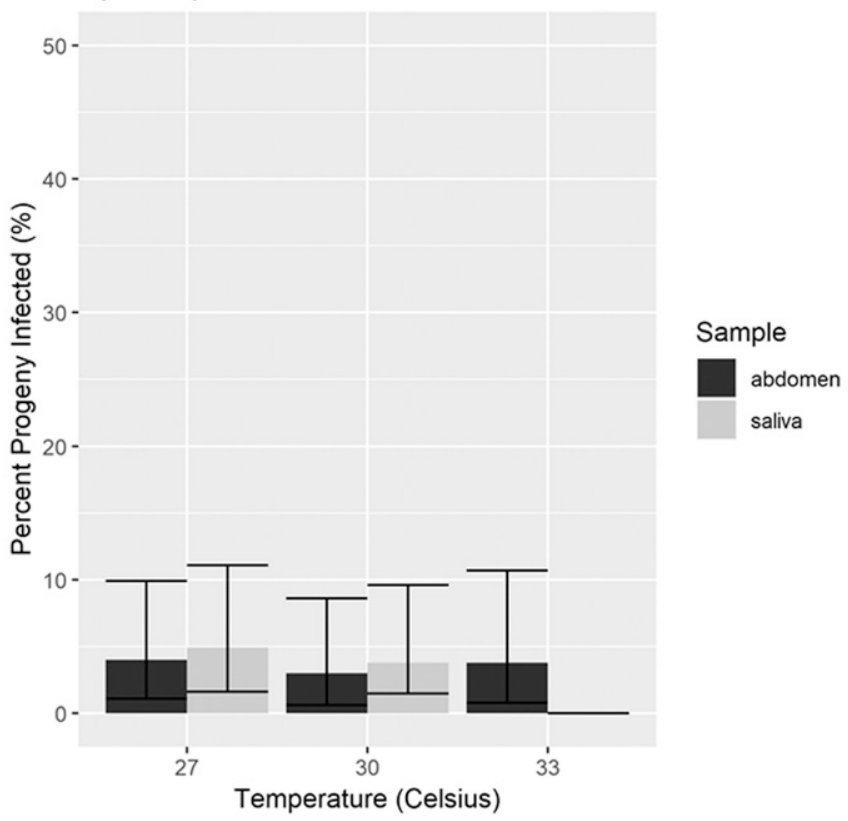

FIGURE 1. Vertical transmission by female temperature and sample type. (A) Percent of vertically infected progeny from each female temperature condition. Vertical transmission occurred to $8.3 \%(n=109,95 \% \mathrm{Cl}=3.8-15.1)$ of progeny from $27^{\circ} \mathrm{C}, 5.7 \%(n=105,95 \% \mathrm{Cl}=2.1-12)$ from $30^{\circ} \mathrm{C}$, and 3.8\% $(n=79,95 \% \mathrm{Cl}=0.8-10.7)$ from $33^{\circ} \mathrm{C}$. (B) Percent of progeny with Zika virus (ZIKV) present in abdomens, indicating vertical infection, and saliva, indicating potential infectiousness, from each female temperature condition. Zika virus was found in progeny abdomens at every temperature: $4 \%(n=100,95 \% \mathrm{Cl}=1.1-9.9)$ of progeny abdomens from $27^{\circ} \mathrm{C}, 3 \%(n=99,95 \% \mathrm{Cl}=0.6-8.6)$ from $30^{\circ} \mathrm{C}$, and $3.8 \%(n=79,95 \% \mathrm{Cl}=$ $0.8-10.7)$ from $33^{\circ} \mathrm{C}$. Zika virus was found in the saliva of $4.9 \%(n=102,95 \% \mathrm{Cl}=1.6-11.1)$ of progeny from $27^{\circ} \mathrm{C}$ to $3.8 \%(n=104,95 \% \mathrm{Cl}=1.5-9.6)$ from $30^{\circ} \mathrm{C}$, but not in any of the 77 progeny from the $33^{\circ} \mathrm{C}$ treatment. 


\section{ZIKV Vertical Transmission by Gonotrophic Cycle}

\section{A By Individual Progeny}

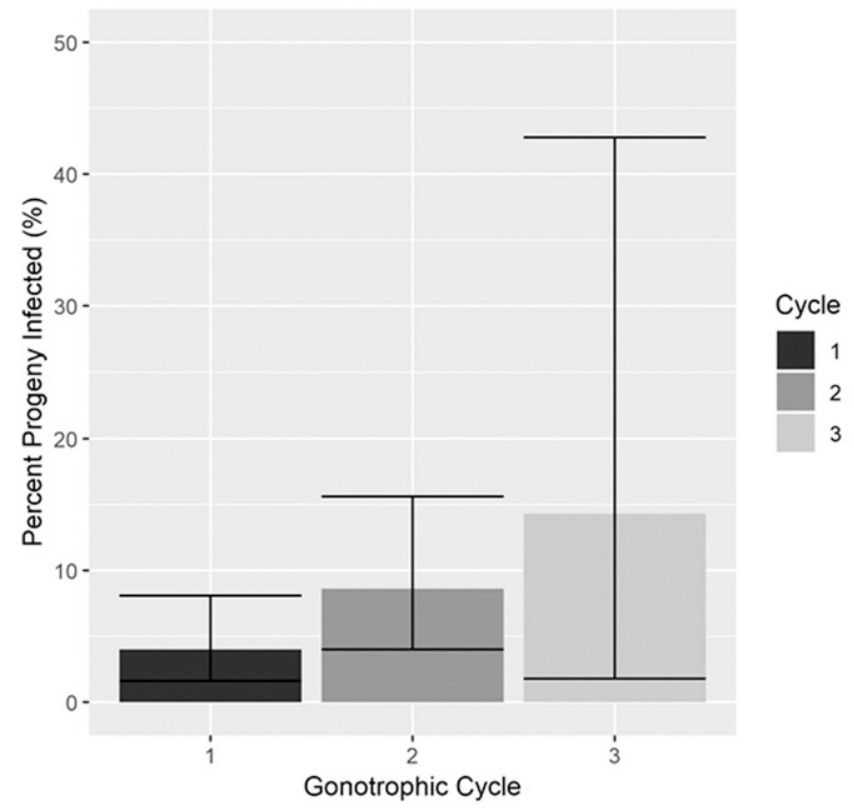

B By Sample

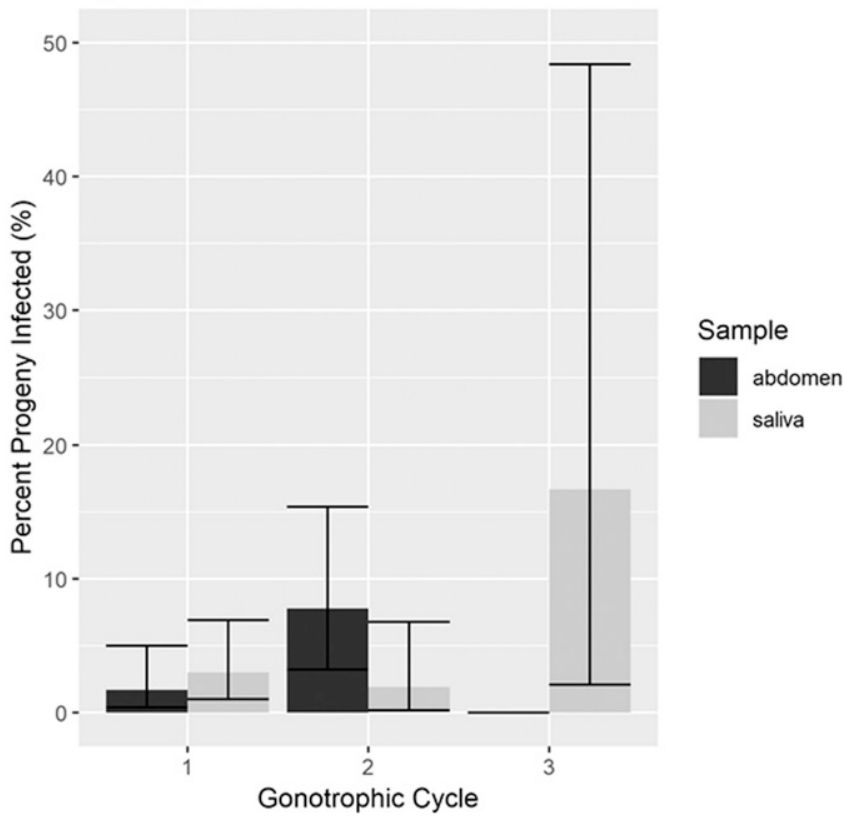

Figure 2. Vertical transmission by gonotrophic cycle and sample type. (A) Percent of vertically infected progeny from each gonotrophic cycle. Zika virus (ZIKV) was detected $4.0 \%(n=174,95 \% \mathrm{Cl}=1.6-8.1)$ of progeny from the first gonotrophic cycle, $8.6 \%(n=105,95 \% \mathrm{Cl}=4-15.6)$ from the second, and $14.3 \%(n=14,95 \% \mathrm{Cl} 1.8-42.8)$ from the third. (B) Percent of progeny with ZIKV present in abdomens, indicating vertical infection, and saliva, indicating potential infectiousness, from each gonotrophic cycle. Zika virus was present in the abdomens of $1.7 \%(n=174,95 \% \mathrm{Cl}=0.4-5.0)$ progeny from the first gonotrophic cycle and $7.8 \%(n=90,95 \% \mathrm{Cl}=3.2-15.4)$ progeny from the second, but not the third. Zika virus was detected in the saliva of $3.0 \%(n=166,95 \% \mathrm{Cl}=1.0-6.9)$ progeny from the first cycle, $1.9 \%(n=104,95 \% \mathrm{Cl}=0.2-6.8)$ of progeny from the second cycle, and $16.7 \%(n=12,95 \% \mathrm{Cl}=2.1-48.4)$ of progeny from the third cycle.

Zika virus vertical transmission occurs during every gonotrophic cycle. To examine vertical transmission over the reproductive lifetime of orally infected female mosquitoes, we measured transmission to progeny over consecutive gonotrophic cycles. Zika virus was vertically transmitted to adult progeny, regardless of whether the maternal mosquito had previously laid eggs (Figure 2A). Zika virus was present in the abdomens of adult progeny from the first and second gonotrophic cycles but was not detected in the abdomens of the 14 surviving progeny from the third gonotrophic cycle. Potentially infectious saliva was collected from adult progeny from all gonotrophic cycles (Figure 2B). The relatively small sample size of the third gonotrophic cycle was due to decreased survivorship and fecundity of female mosquitoes after laying two previous batches of eggs. The gonotrophic cycle significantly affected the odds of overall ZIKV infection in progeny $(P=0.05$, odds ratio =2). However, there was no discernible influence of the gonotrophic cycle on the odds of viral presence in abdominal tissue or saliva samples individually (Table 1).

Zika virus infection persists for at least 2 weeks in infected progeny. Persistence of ZIKV in vertically infected progeny is essential for viral maintenance within mosquito populations and would result in greater opportunities for the virus to be passed to vertebrate hosts during blood feeding. We found ZIKV in progeny as young as 2 days and as old as 2 weeks after adult eclosion. Zika virus was detected in both abdomens and saliva at all ages tested (Figure 3B). Progeny age was significantly associated with reduced odds of viral presence in progeny mosquitoes overall $(P=0.05$, odds ratio $=$ $0.87)$ and in progeny abdomens $(P=0.04$, odds ratio $=0.77)$, but not with viral presence in saliva (Table 1).

Vertically infected progeny have ZIKV in their saliva. For transmission to the vertebrate host to occur, virus must be present in the mosquito's saliva. Zika virus-positive saliva was detected in $3.4 \%(95 \% \mathrm{Cl}=1.6-6.2)$ of progeny, or half of all vertically infected progeny. None of the explanatory variables were significantly associated with ZIKV presence in saliva (Table 1). In addition, viral presence in progeny abdomens was not significantly associated with viral presence in saliva $\left(X^{2}=\right.$ $0.001, P=0.97$ ), and only occurred in one progeny mosquito. Although we did not detect much overlap in the abdomen and saliva of infected individuals, many of the negative samples that came from mosquitoes with detectable ZIKV in another sample bordered our $C_{t}$ cutoff value of 35 . We found the average $C_{t}$ value for nonpositive samples that came from a positive mosquito was 37.88 . In addition, all but six of the nonpositive counterpart samples fell below a $C_{t}$ value of 38 . Our more conservative cutoff of 35 was selected based on the average $C_{t}$ value of our negative controls, which had a mean of $37.74(95 \% \mathrm{Cl}=35.83-39.65)$ across all plates.

\section{DISCUSSION}

Understanding the capacity of ZIKV to establish itself in mosquito populations and persist through periods of low transmission is important to assessing the risk of future 


\section{ZIKV Vertical Transmission by Adult Progeny Age}

A By Individual Progeny

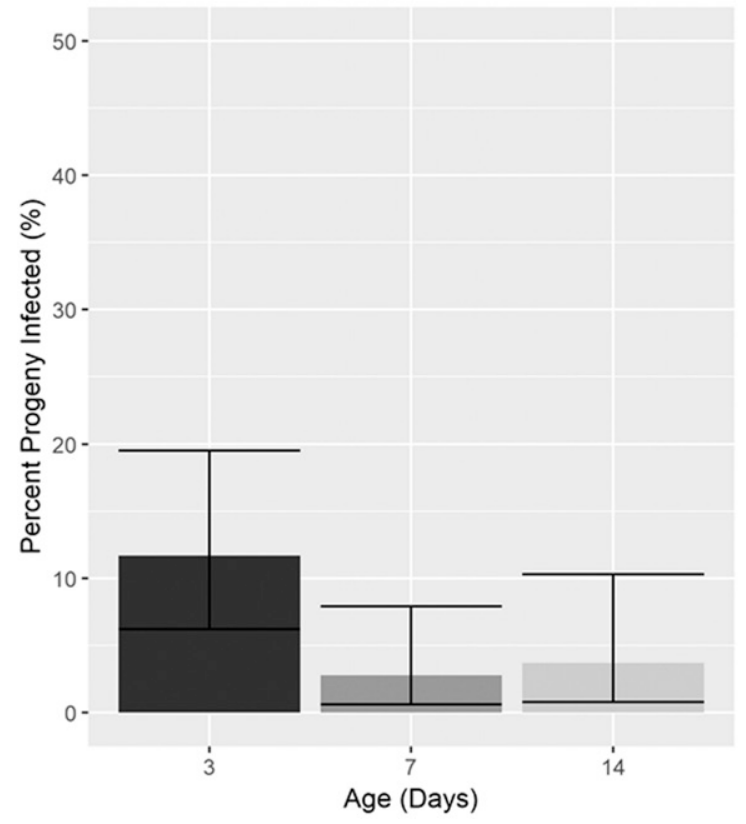

B By Sample

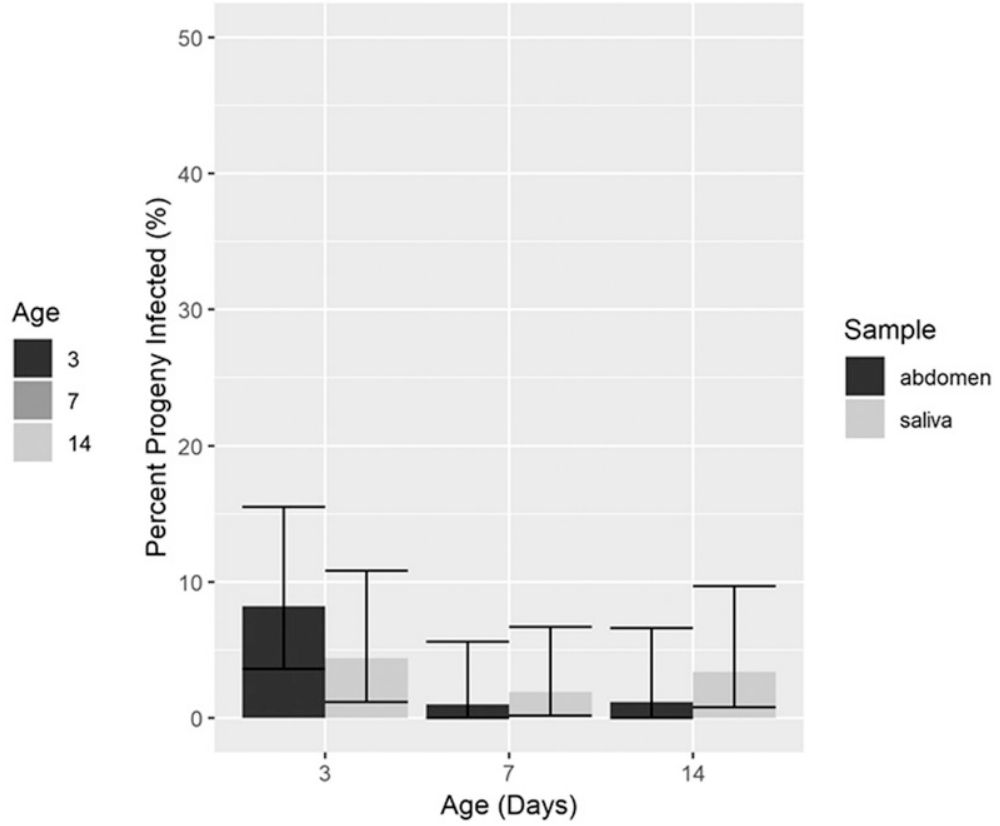

FIGURE 3. Vertical transmission by progeny age and sample type. (A) Percent of vertically infected progeny by age (days) at dissection. Zika virus (ZIKV) was detected in $11.7 \%(n=103,95 \% \mathrm{Cl}=6.2-19.5)$ of 3-day-old progeny, $2.8 \%(n=108,95 \% \mathrm{Cl}=0.6-7.9)$ of 7 -day-old progeny, and $3.7 \%$ $(n=82,95 \% \mathrm{Cl}=0.8-10.3)$ of 14-day-old progeny. (B) Percent of progeny with ZIKV present in abdomens, indicating vertical infection, and saliva, indicating potential infectiousness, by age (days) at dissection. Zika virus was detected in the abdomens of $8.2 \%(n=98,95 \% \mathrm{Cl}=3.6-15.5)$ of 3-day-old progeny, $1.0 \%(n=98,95 \% \mathrm{Cl}=0-5.6)$ of 7-day-old progeny, and $1.2 \%(n=82,95 \% \mathrm{Cl}=0-6.6)$ of 14 -day-old progeny. Zika virus was detected in the saliva of $4.4 \%(n=91,95 \% \mathrm{Cl}=1.2-10.8)$ of 3-day-old progeny, $1.9 \%(n=105,95 \% \mathrm{Cl}=0.2-6.7)$ of 7 -day-old progeny, and $3.4 \%(n=87,95 \% \mathrm{Cl}=0.8-9.7)$ of 14 -day-old progeny.

outbreaks. We found an overall vertical transmission rate of $6.5 \%$ from orally infected female $A$. aegypti to adult female progeny. This proportion is higher than the $0.34 \%$ minimum transmission rate observed in another study of ZIKV vertical transmission in $A$. aegypti, and in the upper range of vertical transmission rates compared with other Aedes-borne arboviruses, including dengue (0.05-2.7\%), chikungunya $(20.2 \%)$, and yellow fever (0.2-8.2\%). ${ }^{11,12,18,34}$ Although ZIKV-positive males have been identified in Mexico and Brazil, potentially corroborating its occurrence in the field, vertical transmission alone is likely insufficient to cause future outbreaks. ${ }^{35,36}$ The estimated minimum rate of vertical transmission required to influence human cases of a similar arbovirus, such as dengue virus, ranges from $4 \%$ to $20-30 \% .^{37,38}$ Some studies have contested that human movement and asymptomatic cases contribute more significantly to human dengue prevalence than vertical transmission. ${ }^{38,39}$ However, peaks in the vertical transmission of dengue have been shown to precede disease outbreaks during periods of high mosquito density. ${ }^{40}$ In light of the rate of vertical transmission observed in this study and others, it is likely vertical transmission that contributes most to ZIKV epidemiology by enabling viral persistence during adverse environmental conditions and interepidemic periods.

Unlike horizontal transmission, the invasion of the midgut by the virus and the invasion of the salivary glands are unlinked because transmission occurs from the mother to eggs rather than from ingesting an infected blood meal. The pathology of ZIKV, including which tissues the virus is present and when, in vertically infected mosquito progeny has not been thoroughly investigated. We detected virus in both abdomens and saliva of vertically infected adult progeny. Although we cannot confirm through plaque assay the presence of active virus, as entire samples were processed for use during qPCR assays, it is unlikely that inactive, fragmented virus would persist both across generations and post-eclosion in infected adult progeny. Although we did not detect much overlap in the abdomen and saliva of infected individuals, many of the negative samples that came from mosquitoes with detectable ZIKV in another sample bordered our $\mathrm{C}_{t}$ cutoff value of 35 and decreased below a $\mathrm{C}_{t}$ value of 38 , the threshold value used by the CDC for ZIKV detection. Although this decreases the sensitivity of our analyses, we prioritized minimizing false positives. Of course, this conservative cutoff also means that some positive tissues, such as abdomens in saliva-positive mosquitoes, were scored as negatives.

We detected vertical transmission of ZIKV from infected females to all egg batches, regardless of previous parity. Although vertical transmission is generally lower in the first gonotrophic cycle for arthropod-borne viruses, it does occur. ${ }^{7}$ Biologically, this may be because the virus has had more time to spread to the mosquito's reproductive system in later gonotrophic cycles. The greater maternal viral infection and dissemination rates in later gonotrophic cycles reflect results of previous studies, whereas the presence of female mosquitoes with disseminated infection at the time of the first gonotrophic cycle supports our detection of vertical 
transmission. Although we found no significant difference between gonotrophic cycles in our analysis, the lower fecundity of female mosquitoes in the third gonotrophic cycle, and thus smaller sample size, may have reduced our power to detect a significant association. Orally infected $A$. aegypti females were twice as likely to vertically transmit ZIKV to their progeny with each consecutive gonotrophic cycle $(P=0.05$, odds ratio $=2$ ). However, analyses considering abdomen and salivary infections separately did not find a significant association between the gonotrophic cycle and progeny infection (Table 1), which could have arisen because of reduced statistical power due to the low number of progeny infected overall. Mosquito survivorship in the field is significantly lower than that under laboratory conditions, and even in the laboratory, not many female mosquitoes lived to complete a third gonotrophic cycle, as reflected by our small sample size. ${ }^{17}$ Accordingly, the shorter life span of mosquitoes in the field may attenuate the impact of higher transmission rates during later gonotrophic cycles. The fact that vertical infection can occur during any gonotrophic cycle, coupled with the prolonged desiccation resistance of $A$. aegypti eggs, means that vertical transmission may provide a mechanism for ZIKV to overwinter in the mosquito population. ${ }^{14}$

The presence of virus in the saliva of vertically infected progeny provides ZIKV with a potential link between vertical and horizontal transmission. This link was present at all progeny ages tested, with ZIKV detected in the saliva as early as 3 days and as late as 14 days after adult emergence. It was interesting to note that virus prevalence was greatest in 3-day-old mosquitoes and dropped in 7- and 14-day-old females. This is in contrast to mosquitoes that acquire the virus horizontally, where viral titers typically increase with time. ${ }^{16,41}$ Vertically infected mosquitoes bypass two essential requirements necessary for horizontal transmission, obtaining the initial infectious blood meal and surviving the EIP. The female mosquitoes in our study had a minimum EIP of 3 days, ${ }^{42}$ and other studies found the average ZIKV EIP to range from 4 to 10 days. ${ }^{15,16}$ Without the need to consume an initial infectious blood meal and survive the EIP, a greater proportion of young mosquitoes are potentially capable of ZIKV. Whether a short incubation period between adult emergence and competent transmission exists for vertically infected progeny and whether there is an effective difference in vector competence between vertically and horizontally infected mosquitoes should be the subject of further research.

\section{CONCLUSION}

We determined that vertical transmission from orally infected females occurs as early as the first reproductive cycle following the initial infectious blood meal at a range of temperatures, and ZIKV persists in the infected progeny for at least 2 weeks after adult eclosion. Virus was detected in the saliva of vertically infected progeny, and the resulting reduction or even elimination of the EIP means these mosquitoes have a longer time frame to bite and infect a human than horizontally infected mosquitoes. Consequently, this work indicates the virus may be maintained in mosquito populations without a vertebrate host for short periods, facilitating ZIKV persistence while retaining a path back to the human host.

Received September 19, 2019. Accepted for publication May 4, 2020.

Published online June 8, 2020.
Acknowledgments: We would like to extend our heartfelt thanks to Teresa Joy, for her assistance in the laboratory; Michael Pham, for his cell culture expertise; and Jenet Soto-Shoumaker, for maintaining the large research colonies that made this work possible. We would also like to thank Kathryn Fitzpatrick for her troubleshooting advice.

Financial support: This work was supported by an NSF Rapid award (1641029) and an NSF Graduate Research Fellowship (DGE-1746060) to Genevieve Comeau.

Dedication: All of the authors would like to dedicate this work to $\mathrm{Dr}$. Genevieve Comeau, a brilliant young scientist and vector biologist who we lost too soon. Her leadership throughout the entire project is reflected in this work and is but one piece of her scholarship. Her passion for science, talent, and dedication to community engagement are a loss to us all.

Authors' addresses: Genevieve Comeau, Taylor Scott, Kathleen Walker, Yves Carrière, and Michael A. Riehle, Department of Entomology, University of Arizona, Tucson, AZ, E-mails: genvcomeau@gmail.com, tys@ email.arizona.edu, krwalker@cals.arizona.edu, ycarrier@ag.arizona.edu, and mriehle@ag.arizona.edu. Robert A. Zinna, Department of Biology, Mars Hill University, Mars Hill, NC, E-mail: robert_zinna@mhu.edu. Kacey Ernst, Department of Epidemiology and Biostatistics, Mel and Enid Zuckerman College of Public Health, University of Arizona, Tucson, AZ, E-mail: kernst@email.arizona.edu.

\section{REFERENCES}

1. Weaver SC, Costa F, Garcia-Blanco MA, Ko Al, Ribeiro GS, Saade G, Shi PY, Vasilakis N, 2016. Zika virus: History, emergence, biology, and prospects for control. Antivir Res 130: 69-80.

2. Soares $C N$, Brasil $P$, Carrera RM, Sequeira $P$, De Filippis $A B$, Borges VA, Theophilo F, Ellul MA, Solomon T, 2016. Fatal encephalitis associated with Zika virus infection in an adult. $J$ Clin Virol 83: 63-65.

3. Dasgupta S, 2016. Patterns in Zika virus testing and infection, by report of symptoms and pregnancy status-United States, January 3-March 5, 2016. MMWR Morb Mortal Wkly Rep 65: 395-399.

4. Tham H, Balasubramaniam V, Ooi MK, Chew M, 2018. Viral determinants and vector competence of Zika virus transmission. Front Microbiol 9: 1040.

5. Alaniz AJ, Bacigalupo A, Cattan PE, 2017. Spatial quantification of the world population potentially exposed to Zika virus. Int $J$ Epidemiol 46: 966-975.

6. Weaver SC, Reisen WK, 2010. Present and future arboviral threats. Antivir Res 85: 328-345.

7. Lequime S, Paul RE, Lambrechts L, 2016. Determinants of arbovirus vertical transmission in mosquitoes. PLOS Pathog 12: e1005548.

8. Nasci RS, Savage HM, White DJ, Miller JR, Cropp BC, Godsey MS, Kerst AJ, Bennett P, Gottfried K, Lanciotti RS, 2001. West Nile virus in overwintering Culex mosquitoes, New York city, 2000. Emerg Infect Dis 7: 742-744.

9. Dhileepan K, Azuolas J, Gibson C, 1996. Evidence of vertical transmission of Ross River and sindbis viruses (Togaviridae: Alphavirus) by mosquitoes (Diptera: Culicidae) in southeastern Australia. J Med Entomol 33: 180-182.

10. Kow CY, Koon LL, Yin PF, 2001. Detection of dengue viruses in field caught male Aedes aegypti and Aedes albopictus (Diptera: Culicidae) in Singapore by type-specific PCR. J Med Entomol 38: 475-479.

11. Agarwal A, Dash PK, Singh AK, Sharma S, Gopalan N, Rao PVL, Parida MM, Reiter P, 2014. Evidence of experimental vertical transmission of emerging novel ECSA genotype of chikungunya virus in Aedes aegypti. PLoS Negl Trop Dis 8: e2990.

12. Fontenille D, Diallo M, Mondo M, Ndiaye M, Thonnon J, 1997. First evidence of natural vertical transmission of yellow fever virus in Aedes aegypti, its epidemic vector. Trans $R$ Soc Trop Med Hyg 91: 533-535.

13. Baqar S, Hayes CG, Murphy JR, Watts DM, 1993. Vertical transmission of West Nile virus by Culex and Aedes species mosquitoes. Am J Trop Med Hyg 48: 757-762. 
14. Sota T, Mogi M, 1992. Interspecific variation in desiccation survival time of Aedes (stegomyia) mosquito eggs is correlated with habitat and egg size. Oecologia 90: 353-358.

15. Wong PJ, Li MI, Chong C, Ng L, Tan C, 2013. Aedes (stegomyia) albopictus (skuse): a potential vector of Zika virus in Singapore. PLoS Negl Trop Dis 7: e2348.

16. Roundy $\mathrm{CM}$ et al., 2017. Variation in Aedes aegypti mosquito competence for Zika virus transmission. Emerg Infect Dis 23: 625-632.

17. Brady OJ, Johansson MA, Guerra CA, Bhatt S, Golding N, Pigott DM, Delatte H, Grech MG, Leisnham PT, Maciel-de-Freitas R, 2013. Modelling adult Aedes aegypti and Aedes albopictus survival at different temperatures in laboratory and field settings. Parasites Vectors 6: 351.

18. Thangamani S, Huang J, Hart CE, Guzman H, Tesh RB, 2016. Vertical transmission of Zika virus in Aedes aegypti mosquitoes. Am J Trop Med Hyg 95: 1169-1173.

19. Ciota AT, Bialosuknia SM, Ehrbar DJ, Kramer LD, 2017. Vertical transmission of Zika virus by Aedes aegypti and Ae. albopictus mosquitoes. Emerg Infect Dis 23: 880-882.

20. Li C, Guo X, Deng Y, Xing D, Sun A, Liu Q, Wu Q, Dong Y, Zhang Y, Zhang $\mathrm{H}, 2017$. Vector competence and transovarial transmission of two Aedes aegypti strains to Zika virus. Emerg Microbes Infect 6: 1-7.

21. Tran A, L'Ambert G, Lacour G, Benoît R, Demarchi M, Cros M, Cailly P, Aubry-Kientz M, Balenghien T, Ezanno P, 2013. A rainfall-and temperature-driven abundance model for Aedes albopictus populations. Inter J Environ Res Public Health 10: 1698-1719.

22. Focks DA, Haile D, Daniels E, Mount GA, 1993. Dynamic life table model for Aedes aegypti (Diptera: Culicidae): analysis of the literature and model development. I Med Entomol 30: 1003-1017.

23. Costa EAPdeA, Santos EMdeM, Correia JC, Albuquerque CMRde, 2010. Impact of small variations in temperature and humidity on the reproductive activity and survival of Aedes aegypti (Diptera, Culicidae). Rev Bras Entomol 54: 488-493.

24. Barbazan P, Guiserix M, Boonyuan W, Tuntaprasart W, Pontier D, Gonzalez J, 2010. Modelling the effect of temperature on transmission of dengue. Med Vet Entomol 24: 66-73.

25. da Costa CF et al., 2018. Evidence of vertical transmission of Zika virus in field-collected eggs of Aedes aegypti in the Brazilian Amazon. PLoS Negl Trop Dis 12: e0006594.

26. Izquierdo-Suzan M, Zarate S, Torres-Flores J, Correa-Morales F, Gonzalez-Acosta C, Sevilla-Reyes EE, Lira R, Alcaraz-Estrada SL, Yocupicio-Monroy M, 2019. Natural vertical transmission of Zika virus in larval Aedes aegypti populations, Morelos, Mexico. Emerg Infect Dis 25: 1477-1484.

27. Ndoen E, Wild C, Dale P, Sipe N, Dale M, 2012. Mosquito longevity, vector capacity, and malaria incidence in West Timor and Central Java, Indonesia. ISRN Public Health 2012: 1-5.
28. Klowden MJ, Lea AO, 1978. Blood meal size as a factor affecting continued host-seeking by Aedes aegypti (L.). Am J Trop Med Hyg 27: 827-831.

29. Black IVWC, Bennett KE, Gorrochótegui-Escalante N, BarillasMury CV, Fernández-Salas I, de Lourdes Muñoz M, Farfán-Alé JA, Olson KE, Beaty BJ, 2002. Flavivirus susceptibility in Aedes aegypti. Arch Med Res 33: 379-388.

30. Agbulos DS, Barelli L, Giordano BV, Hunter FF, 2016. Zika virus: quantification, propagation, detection, and storage. Curr Protoc Microbiol 43: 15D.4.1-15D.4.16.

31. Franz A, Kantor A, Passarelli A, Clem R, 2015. Tissue barriers to arbovirus infection in mosquitoes. Viruses 7: 3741-3767.

32. Center for Disease Control and Prevention, 2017. Trioplex Real Time PCR Assay: Instructions for Use. Available at: https:// www.cdc.gov/Zika/pdfs/trioplex-real-time-rt-pcr-assayinstructions-for-use.pdf. Accessed May 25, 2020.

33. Gallian P, Cabie A, Richard P, Paturel L, Charrel RN, Pastorino B, Leparc-Goffart I, Tiberghien P, de Lamballerie X, 2017. Zika virus in asymptomatic blood donors in Martinique. Blood 129: 263-266.

34. Joshi V, Mourya D, Sharma R, 2002. Persistence of dengue-3 virus through transovarial transmission passage in successive generations of Aedes aegypti mosquitoes. Am J Trop Med Hyg 67: 158-161.

35. Elizondo-Quiroga D, Medina-Sanchez A, Sanchez-Gonzalez JM, Eckert KA, Villalobos-Sanchez E, Navarro-Zuniga AR, Sanchez-Tejeda G, Correa-Morales F, Gonzalez-Acosta C, Arias CF, 2018. Zika virus in salivary glands of five different species of wild-caught mosquitoes from Mexico. Sci Rep 8: 809.

36. Ferreira-de-Brito A, Ribeiro IP, Miranda RM, Fernandes RS, Campos SS, Silva KA, Castro MG, Bonaldo MC, Brasil P, Lourenço-de-Oliveira $R, 2016$. First detection of natural infection of Aedes aegypti with Zika virus in Brazil and throughout South America. Mem Inst Oswaldo Cruz 111: 655-658.

37. DeFoliart G, Grimstad P, Watts D, 1987. Advances in mosquitoborne arbovirus/vector research. Annu Rev Entomol 32: 479-505.

38. Adams $B$, Boots $M, 2010$. How important is vertical transmission in mosquitoes for the persistence of dengue? Insights from a mathematical model. Epidemics 2: 1-10.

39. Grunnill M, Boots $M, 2015$. How important is vertical transmission of dengue viruses by mosquitoes (Diptera: Culicidae)? J Med Entomol 53: 1-19.

40. Rohani A, Wong YC, Zamre I, Lee HL, Zurainee MN, 2009. The effect of extrinsic incubation temperature on development of dengue serotype 2 and 4 viruses in Aedes aegypti (L.). Southeast Asian J Trop Med Public Health 40: 942-950.

41. Winokur OC, Main BJ, Nicholson J, Barker CM, 2020. Impact of temperature on the extrinsic incubation period of Zika virus in Aedes aegypti. PLoS Negl Trop Dis 14: e0008047.

42. Zinna R, 2018. Zika Extrinsic Incubation Period Data. Available at: https://github.com/Zinnar/Zika_eip. Accessed May 25, 2020. 